\title{
An all-printed wireless humidity sensor label
}

\author{
Xiaodong Wang, Oscar Larsson, Duncan Platt, Staffan Nordlinder, Isak Engquist, \\ Magnus Berggren and Xavier Crispin
}

\section{Linköping University Post Print}

N.B.: When citing this work, cite the original article.

Original Publication:

Xiaodong Wang, Oscar Larsson, Duncan Platt, Staffan Nordlinder, Isak Engquist, Magnus Berggren and Xavier Crispin, An all-printed wireless humidity sensor label, 2012, Sensors and actuators. B, Chemical, (166-167), , 556-561.

http://dx.doi.org/10.1016/j.snb.2012.03.009

Copyright: Elsevier

http://www.elsevier.com/

Postprint available at: Linköping University Electronic Press

http://urn.kb.se/resolve?urn=urn:nbn:se:liu:diva-79036 


\section{An all-printed wireless humidity sensor label}

Xiaodong Wang ${ }^{\mathrm{a}}$, Oscar Larsson ${ }^{\mathrm{a}}$, Duncan Platt ${ }^{\mathrm{b}}$, Staffan Nordlinder ${ }^{\mathrm{c}}$, Isak Engquist ${ }^{\mathrm{a}, \star}$, Magnus Berggren ${ }^{a}$, Xavier Crispin $^{a}$

${ }^{a}$ Dept. of Science and Technology (ITN), Linköping University, SE-601 74, Norrköping, Sweden

${ }^{\mathrm{b}}$ Acreo AB, Box 787, SE-601 17, Norrköping, Sweden

${ }^{c}$ WebShape AB, Box 787, SE-601 17, Norrköping, Sweden

\section{Abstract}

Printed electronics promise various kinds of sensor circuit labels, for applications in distributed sensing and monitoring, which can be manufactured using traditional printing tools at very low cost. Elevated humidity levels or water leakages cause tremendous costs in our society, such as in construction industries and in transportations. Distributed monitoring and remote sensing of the humidity level inside walls of buildings and packages is therefore desired and urgently needed. Here, we report a wireless humidity sensor label that is manufactured using screen-printing and dry-phase patterning. The sensor label includes a planar antenna, a tuning capacitor and a printed sensor-capacitor head. Through electromagnetic coupling between a reader and the printed sensor label, changes in humidity level were remotely detected and read-out as a shift of the resonant frequency. The manufacturing process of the humidity sensor label is fully compatible with inexpensive, reelto-reel processing technologies, thus enabling low cost production.

\section{Keywords:}

Printed humidity sensor label

Wireless sensor

Polyelectrolyte

Dry-phase patterning

Resonance 


\section{Introduction}

In a world where electronic devices are everywhere and our demands on quality and effectiveness are always increasing, there is a need to develop cheap, distributed and reliable sensors in order to monitor different environmental parameters, such as humidity [1, 2], temperature [3], gas concentration [4-6], $\mathrm{pH}$ [7] etc. One challenging task is to utilize sensor systems to monitor parameters in sealed environments, such as in food and pharmaceutical packages, inside an animal, inside construction elements, etc., because a physical connection between the sensor and the data processing unit is often difficult, or in some cases even impossible, to establish. In such applications, wireless and remote sensing systems are of interest. Several remote sensor concepts have been explored and are in use today, among which combining conventional electronic sensor components with RFID technology is one option [8,9]. Although the technology for such remote and distributed sensors matures very rapidly today, wireless RFID sensors that are integrated into packages and various construction materials are rare. The main hurdle for a widespread use of RFID sensors in distributed items is the high cost to manufacture included components and to assemble the final RFID sensor tag. An interesting alternative to this batch-based and expensive production scheme is found in large area and/or reel-to-reel printing and patterning technology developed for the graphic art and printed media industry. Some of these techniques are actually suited for manufacturing sensors combined with resonance circuits and antennas, which defines a chipless RFID sensor system.

Moisture is one of the largest concerns for the construction and building industry today. In Sweden alone, the estimated annual cost for combating moisture-related problems in houses and buildings exceeds 300 million Euros. A significant part of this cost arises because the problems are detected too late, when mould damage has already occurred. Further, the present cumulated value of these escalating problems is estimated to exceed 10 billion Euros [10]. Thus, there is a considerable need for a low-cost wireless humidity sensor that can be distributed and embedded into construction materials. Different active materials 
such as ceramics [11] and composites [12] have been investigated to sense and monitor humidity changes. One promising material system for the above-mentioned applications is polyelectrolytes. These materials, in which polar groups are covalently attached to a polymeric backbone, are promising candidates since they are normally hygroscopic and express major changes in their impedance characteristics upon exposure to different humidity levels [13-15]. Various mechanisms govern the membrane charge polarization characteristics in polyelectrolyte thin films [16]. The polyelectrolytes can dissociate into mobile ions and immobile polymer counter-ions and the ionic migration characteristic is dramatically affected by the degree of water absorption, i.e. humidity sensitive. Moreover, polyelectrolytes are easily printable using standard printing techniques [17].

Here, we report the properties of a stand-alone printed sensor head based on a polyelectrolyte derivative. We exploit ionic migration and polarization within the polyelectrolyte in the frequency region below $1 \mathrm{MHz}$ in order to monitor changes in relative humidity. This sensor head structure is then used in a fully printed humidity sensor label also comprising an antenna and a tuning capacitor, all produced using printing processes. An external reader powers the resulting printed sensor label through electromagnetic induction. The sensor readout is communicated through the resonant frequency of the printed sensor label that alters depending on the different ambient humidity levels. Since the printed sensor label is passive, i.e. does not include any battery, it is expected to have a long life-time and can be used as a distributed sensor label in a vast array of applications. Further, reel-to-reel manufacturability enables low-cost production at very high volumes.

Fig. 1 (a) displays a function block diagram of the printed sensor label, which consists of three functional components: a planar antenna, a tuning capacitor, and the sensor component. In our design [18], the printed sensor head is connected to an LC resonance circuit which consists of the tuning capacitor and the planar antenna. The tuning capacitor is necessary in order to shift the resonant frequency of the printed sensor label into the desired frequency band. Fig. 1 (b) gives the electrical equivalent circuit. The inductance 
of the planar antenna is represented by $L_{\text {antenna }}$. $C_{\text {tuning }}$ represents the capacitance of the tuning capacitor and $Z_{\text {sensor }}$ is the total impedance of the printed sensor head.

Fig.1

When a reader antenna is positioned in close proximity to the printed sensor label, an impedance signature from the label is reflected back to the reader via electromagnetic coupling. The reflected impedance, $Z_{\text {reflected, }}$ is given by [19]

$Z_{\text {reflected }}=\frac{\omega^{2} M^{2}}{Z_{\text {label }}}$

where $\omega$ is the angular frequency and $M$ is the mutual inductance from the electromagnetic coupling between the reader antenna and the printed sensor label antenna. $Z_{\text {label }}$ is the total impedance of the printed sensor label under electromagnetic coupling, which is given by

$$
\begin{aligned}
& Z_{\text {label }}=\frac{C_{\text {sensor }}^{2} R_{\text {sensor }}}{\left(\omega C_{\text {tuning }} C_{\text {sensor }} R_{\text {sensor }}\right)^{2}+\left(C_{\text {tuning }}+C_{\text {sensor }}\right)^{2}} \\
& +j\left[\omega L_{\text {antenna }}-\frac{C_{\text {tuning }}+C_{\text {sensor }}+\omega^{2} C_{\text {tuning }} C_{\text {sensor }}^{2} R_{\text {sensor }}^{2}}{\omega^{3} C_{\text {tuning }}^{2} C_{\text {sensor }}^{2} R_{\text {sensor }}^{2}+\omega\left(C_{\text {tuning }}+C_{\text {sensor }}\right)^{2}}\right]
\end{aligned}
$$

The resonant frequency is referred to as the frequency at which the total impedance reaches a minimum in case of a series LC circuit or a maximum in case of a parallel LC circuit. Since $Z_{\text {reflected }}$ is inversely proportional to $Z_{\text {label }}$, any change in the impedance of the printed sensor label leads to a corresponding modification of the impedance within the reader circuit. This means that the resonant frequency of the reader circuit is dependent on the impedance of the printed sensor head and thus also on the moisture level. Hence, by measuring the resonant frequency of the reader circuit, wireless readout of the printed sensor label is accomplished. 


\section{Experimental}

\subsection{Material preparation}

The polyelectrolyte printing solution was prepared as follows: 6 wt $\%$ poly(styrene sulfonic acid) sodium salt (PSSNa, see Fig. 3) with a molecular weight 500,000 g/mol (Alfa Aesar), was dissolved in a mixture of deionized water and isopropanol (>99.7\%, SigmaAldrich). The solution was then put into an ultrasonic bath for 1 hour at room temperature in order to completely dissolve the solute. The printable PSSNa solution has a viscosity of 70 $\mathrm{mPa} \cdot \mathrm{S}$ at $21^{\circ} \mathrm{C}$ (A\&D SV-10 vibro viscometer). Printable dielectric polymeric composition 5018, conductive silver paste 5000 and conductive carbon composition 7102 were used as received from DuPont.

The planar antenna $(100 \times 100 \mathrm{~mm})$ of the printed sensor label, see Fig. 2 (a), was produced, via a dry-phase milling process [20], by the company WebShape AB [21]. The antenna pattern was defined in a $9 \mu \mathrm{m}$ thick laminated aluminum foil. The electric resistance of the antenna pattern is about 42 ohms and its inductance is around $70 \mu \mathrm{H}$. Bottom electrodes for the tuning capacitor (aluminum pads in the dashed frame in Fig. 2 (a)) and the printed sensor head (aluminum pads in the solid frame in Fig. 2 (a)) were fabricated along with the antenna in the same process. The antenna and the bottom electrodes were cleaned by acetone and isopropanol prior to their printing process.

\subsection{Device fabrication}

The sensor label was printed using a flatbed screen printer (TIC SCF 550) under ambient conditions. The bottom electrodes and the antenna, shown in Fig. 2 (a), were cleaned before printing. The electrolyte solution was then printed onto the precleaned bottom electrode (Fig. 2 (b)), followed by drying at $90^{\circ} \mathrm{C}$ for 2 minutes. Dielectric material (DuPont 5018) was deposited through a screen printing mesh to cover the whole antenna and the bottom electrode of the tuning capacitor and partially cover the electrolyte layer (Fig. 2 (c)). This dielectric layer serves not only as a dielectric material for the tuning capacitor but 
also as an encapsulating layer for the antenna. The printed dielectric layer was cured under UV light for a few seconds. A carbon conductive composition (DuPont 7102) was then printed onto the uncovered part of the electrolyte layer (Fig. 2 (d)). The sensor label was then dried at $90^{\circ} \mathrm{C}$ for 2 minutes. Then, a thin silver layer (DuPont 5000) was printed to form the top electrode on top of the dielectric layer. This patterning step completes the production of the tuning capacitor $(\sim 7 \mathrm{nF})$ and also forms a connection bridge between the antenna and the printed sensor head (Fig. 2 (e)). Finally, the resulting printed sensor label was baked at $80^{\circ} \mathrm{C}$ for 60 minutes in order to remove residual solvent in the printed layers. A picture of the final label is given in Fig. 2 (f).

Fig 2

\subsection{Measurements}

All measurements were carried out by placing the sensor label in a climate chamber (ACS $\mathrm{CH}-160$ ) at a constant temperature of $23^{\circ} \mathrm{C}$, starting at low humidity, $10 \% \mathrm{RH}$, and increasing up to $90 \% \mathrm{RH}$. The impedance measurements of the printed sensor head were performed by using an Alpha high-resolution dielectric analyzer (Novcontrol), utilizing a sinusoidal $\mathrm{AC}$ voltage of $0.1 \mathrm{~V}_{\mathrm{rms}}$ in a frequency range from $100 \mathrm{~Hz}$ to $1 \mathrm{MHz}$.

In order to receive signals from the printed sensor label and to measure the resonant frequency, we connected a reader antenna to Agilent's E4407B frequency spectrum analyzer. The frequency was swept from $40 \mathrm{kHz}$ to $800 \mathrm{kHz}$ and the output power was set to $2 \mathrm{~mW}$. The printed sensor label was positioned in front of, and parallel with respect to, the reader antenna at a distance of $3 \mathrm{~mm}$.

All the layers of the printed sensor head were measured by a surface profiler (Veeco Dektak $3 \mathrm{ST})$.

\section{Results and discussions}


In this work, both printed stand-alone sensor heads and printed humidity sensor labels were fabricated. We have used screen printing to pattern several materials (polyelectrolyte, dielectric, conductor) needed to create these devices. Since printing is a non-conventional manufacturing method in electronics, we describe the main printing steps in this result section. (More details on materials and processes are found in the experimental section.)

\subsection{Printed stand-alone sensor head}

The stand-alone sensor heads are built up from a capacitor-like structure, see Fig. 3, and were fabricated on a global aluminum bottom electrode. The humidity sensitive polyelectrolyte layer was first printed on top of the bottom electrode, followed by deposition of the dielectric layer. Silver contact pads were deposited on the dielectric layer in order to lower the contact resistance and carbon contacts were then printed on top of the polyelectrolyte films.

Fig. 3

The impedance spectra of the printed sensor head at different humidity levels are given in Fig. 4 (a-b). As the humidity increases, both the real part and imaginary part of the impedance decreases. This is in accordance with the results from spin-coated humidity sensor heads in [18]. Three different mechanisms, active at different time scales, are identified to explain the variation of the impedance of a thin polyelectrolyte film: dipolar relaxation, ionic migration and electric double layer formation [16]. The phase angle at low frequencies is usually around $-80^{\circ}$ [16], i.e. capacitive behavior. With increasing frequency, the phase angle increases and we define the transition frequency as the frequency where the phase angle becomes larger (i.e. closer to $0^{\circ}$ ) than $-45^{\circ}$ for the first time. The transition frequency is specifically correlated to the humidity level and is indicative of the dominating impedance behavior of the sensor capacitor. When the working frequency is lower than the transition frequency, a capacitive behavior dominates the impedance characteristics of the 
polyelectrolyte-based sensor heads. This capacitive character originates from formation of an electric double layer at the interface between the polyelectrolyte and the electrode. When the polyelectrolyte, PSSNa, is polarized under an electric field, mobile sodium cations $\left(\mathrm{Na}^{+}\right)$ that are dissociated from their polymer counter-ions migrate to the negatively charged electrode. The polyanions (PSS) are regarded as immobile. Above the transition frequency, the sensor head primarily behaves as a resistive-type sensor due to ionic movement in the oscillating electric field $[13,18]$. Therefore, a thin film capacitor based on a polyelectrolyte can be seen as both a capacitive-type and a resistive-type humidity sensor depending on the frequency range in which it is operating.

The transition frequency of the printed sensor head at different relative humidity levels is shown in Fig. 4 (c). The transition frequency is relatively constant up to $60 \% \mathrm{RH}$ and then decreases with higher humidity levels. This behavior is somewhat different compared to our previous results achieved from the non-printed sensor component [18] using Ti electrodes. The non-printed sensor shows a significant increase in the transition frequency already above $50 \% \mathrm{RH}$. One possible reason for this discrepancy is that the carbon electrode (resistivity of DuPont 7102 carbon conductive composition: 5 to $7.5 \times 10^{-4}$ $\Omega \cdot m)$ [22] used in the present work contributes relatively more to the net resistance as compared to the sensor based on titanium (resistivity of titanium: $4.2 \times 10^{-7} \Omega \cdot \mathrm{m}$ ). For nonprinted sensor head [18], $Z_{R e}$ decreases much more than $-Z_{I m}$ with increasing humidity. $\left(Z_{\text {Re }}\right.$ is almost an order of magnitude smaller at $90 \% \mathrm{RH}$ than at $30 \% \mathrm{RH}$.) This means that the capacitive component of the sensor impedance becomes more dominating at higher humidity. (i.e. the transition frequency increases when the humidity level rises.) However, in the printed sensor head, the high resistance in the carbon electrode always dominates in $Z_{R e}$, and a relatively small decrease in $Z_{R e}$ was measured between $30 \% R H\left(Z_{R e}=268 \Omega\right.$ at $200 \mathrm{kHz})$ and $90 \% \mathrm{RH}\left(\mathrm{Z}_{\mathrm{Re}}=218 \Omega\right.$ at $\left.200 \mathrm{kHz}\right)$. Thus, even when humidity increases, the resistive component of the sensor impedance remains dominant whilst the capacitive component of the sensor impedance becomes even less dominant due to the decrease in - 
$Z_{\mathrm{Im}}$. This results in a decrease of the transition frequency with increasing humidity. For instance, the printed sensor head has a transition frequency around $70 \mathrm{kHz}$ below $60 \% \mathrm{RH}$, but it reduces down to about $50 \mathrm{kHz}$ above $75 \% \mathrm{RH}$. As a result, a printed sensor head, working between $150 \mathrm{kHz}$ and $250 \mathrm{kHz}$, can always be considered as being primarily a resistive-type sensor.

Fig. 4

\subsection{Printed humidity sensor label}

Fig. 5 (a) shows the evolution of the reader signal with increasing humidity. The resonant frequency at a particular humidity for each measured curve was determined by identifying the power-dip versus the frequency (marked by a larger solid symbol on the graph). As a comparison, a simple calculation assuming an LC resonance circuit $(\mathrm{L}=70 \mu \mathrm{H}$ and $\mathrm{C}=7 \mathrm{nF}$ ) predicts that the printed sensing circuit should resonate at around $230 \mathrm{kHz}$.

Clearly, there is a continuous decrease in the resonant frequency with increasing humidity. The results presented in Fig. 5 (b) indicate a nearly linear sensitivity of the resonant frequency versus humidity of around $-1.1 \mathrm{kHz}$ per $\% \mathrm{RH}$. The printed sensor label responded quickly during water absorption (a few seconds) but relatively slower during desorption (more than 30 minutes to reach a stable reading at $10 \% \mathrm{RH}$ ). This means that the sensor response will show considerable hysteresis for fast changes in humidity levels. In accordance with this, we also observed that extended drying of the printed sensor label (1 hour at $10 \% \mathrm{RH})$ resulted in an even higher resonant frequency $(\sim 270 \mathrm{kHz})$ compared to the initial measured value $(\sim 240 \mathrm{kHz})$ that was recorded after 10 minutes at $10 \% \mathrm{RH}$.

Fig. 5

Similar responses to changes in humidity level were observed both in repeated measurements on the same sample (after 2 months storage) and for several replicated samples. The largest measured deviation from the data in Figure 5, at any $\mathrm{RH}$ value, is 
around $24 \mathrm{kHz}$. We believe the variations are mainly due to the low signal-to-noise ratio at the resonance peak, which originates from the low quality factor in the label $(Q \sim 2)$ and the simple construction of the reading circuit.

\section{Conclusions and future work}

In this paper, we demonstrate an all-printed wireless humidity sensor label which is compatible with low-cost and high-volume printing processes. Using the polyelectrolyte PSSNa as humidity sensing material and carbon as top electrode, the printed sensor head is working as a resistive-type sensor between $150 \mathrm{kHz}$ and $250 \mathrm{kHz}$. Wireless reading is enabled by electromagnetic coupling between the printed sensor label and a reader antenna. The printed sensor label shows a distinct frequency shift of about $80 \mathrm{kHz}$ between dry condition $(10 \% \mathrm{RH})$ and high humidity $(90 \% \mathrm{RH})$. The resonant frequency decreases continuously with increasing humidity, which makes this sensor label a good candidate for wireless humidity measurements. In combination with the potential for low-cost production, we foresee application areas such as monitoring wet/dry conditions behind the walls of a building, or under a floor, where moisture is difficult to detect by other means.

In order to achieve a good discrimination and an acceptable reading distance (10 to $20 \mathrm{~cm}$ ), a high enough quality factor ( $Q$ value) of the printed sensor label is necessary. The $Q$ value in our present work was below 2 because of high resistance in the sensor antenna. To resolve this, working in a higher allowed frequency band, e.g. $3.155 \mathrm{MHz}-3.4 \mathrm{MHz}$, will be one alternative since the sensor antenna then can be redesigned to a smaller size with significantly lower resistance. Investigations of this concept are currently ongoing, together with the development of a more precise resonant frequency measurement method.

\section{Acknowledgements}


The authors wish to acknowledge Brains and Bricks, FORMAS, SSF, VINNOVA and Tillväxtverket for the financial support of this project. We also wish to thank Marie Nilsson and Lars-Olov Hennerdal at Acreo AB for fruitful discussions concerning printing processes.

\section{Biographies}

Xiaodong Wang is a PhD student in Organic Electronics at the Department of Science and Technology, Linköping University, Sweden. He received his M.Sc. in Electrical Engineering at Linköping University in 2009.

Oscar Larsson is a research scientist at Swedish National Laboratory of Forensic Science (SKL). He received his M.Sc. in Electronics in 2004 and his PhD in organic electronics in 2011, both degrees from Linköping University, Sweden.

Duncan Platt received his B.Sc. with 1st class honours in 1986 from the University of Strathclyde, Scotland. His career began working with optical and vibratory gyroscope technology development at GEC Ferranti in Edinburgh. In 1994 he joined Ericsson Telecom in Norrköing, Sweden, working as an electronic design engineer. From 1997 to 2008 he was a consultant at the Swedish company ÅF. Since 2008 he is currently employed as a senior project manager in the Nanoelectronics Department at Acreo. Duncan is a member of the IEEE.

Staffan Nordlinder received his M. Sc. in Applied Physics and Electrical Engineering 2003, Linköping University, Sweden. Staffan has a background as a developer of control systems and electrical design within the paper and pulp industry. After a period of working in the commercial industry he received a M. Sc. with special focus on information and control systems. Since 2002 Staffan has been working at Acreo AB as a scientist and project leader within the area of organic and printed electronics, with the main focus towards the developments of components and production processes for R2R manufacturing. 
Isak Engquist is an assistant professor in Organic Electronics at Linköping University, Sweden. He received his PhD in Applied Physics at Linköping University in 1996, then worked as a research scientist at the Swedish Defence Research Agency and as a senior researcher at Thin Film Electronics AB, before joining the University in 2007. His current research activities include printed organic transistors and sensor circuits. He has published 17 papers in scientific journals and is a co-inventor of 7 patents.

Magnus Berggren is a professor in Organic Electronics at the Department of Science and Technology, Linköping University, Sweden, since 2002. He received his M.Sc. in Physics in 1991 and his PhD in Applied Physics in 1996, both degrees from Linköping University. From 1996 to 1997 he was the managing director of Thin Film Electronics AB and from 1997 to 2001 he was a project manager at Acreo institute. Today, he is guiding a research group of around 20 people at Linköping University aiming at developing novel printed electronics and organic bioelectronics.

Xavier Crispin is an associate professor in Organic Electronics at the Department of Science and Technology, Linköping University, Sweden, since 2004. He received his M.Sc. in Chemistry in 1995 and his $\mathrm{PhD}$ in Chemistry in 2000, both degrees from the University of Mons-Hainaut in Belgium. From 2000 to 2004, he was an assistant professor in Surface Physics and Chemistry at Linköping University. Today, he is supervising the research focused on solid-state electronics within the group of Professor M. Berggren. 


\section{Figure captions:}

Fig. 1 (a) The function block diagram of the printed sensor label. (b) The electrical equivalent circuit of the printed sensor label.

Fig. 2 (Color online) (a)-(e) Five steps of screen printing of a printed sensor label: (a) Photo of precleaned planar antenna and electrodes. (b) Screen printing of the electrolyte layer (yellow). (c) Screen printing of the dielectric layer (blue). (d) Screen printing of the top electrode of the printed sensor head (black). (e) Screen printing of the top electrode of the tuning capacitor and electrical bridges (light grey). (f) Photograph of an all-printed humidity sensor label.

Fig. 3 The structure of a printed sensor head and a photo showing four sensor heads on a common bottom electrode.

Fig. 4 (Color online) (a) The real part and (b) the imaginary part of the measured impedance of the printed senor head (area: $0.03 \mathrm{~cm}^{2}$ ). (c) The transition frequency versus relative humidity $(\mathrm{RH})$.

Fig. 5 (a) The evolution of the sensor label response at different humidity levels. (b) The resonant frequency of the printed sensor label versus relative humidity $(\mathrm{RH})$. 


\section{References}

[1] S. Akita, H. Sasaki, K. Watanabe, A. Seki, A humidity sensor based on a hetero-core optical fiber, Sens. Actuators B: Chem. 147 (2010) 385-391.

[2] X. Lv, Y. Li, P. Li, M. Yang, A resistive-type humidity sensor based on crosslinked polyelectrolyte prepared by UV irradiation, Sens. Actuators B: Chem. 135 (2009) 581-586.

[3] K. A. A. Makinwa, Smart temperature sensors in standard CMOS, Procedia Engineering 5 (2010) 930-939.

[4] P. Pacher, A. Lex, S. Eder, G. Trimmel, C. Slugovc, E. J. W. List, et al., A novel concept for humidity compensated sub-ppm ammonia detection, Sens. Actuators B: Chem. 145 (2010) 181-184.

[5] T. Nagai, S. Tamura, N. Imanaka, Solid electrolyte type ammonia gas sensor based on trivalent aluminum ion conducting solids, Sens. Actuators B: Chem. 147 (2010) 735-740.

[6] J. W. Jeong, Y. D. Lee, Y. M. Kim, Y. W. Park, J. H. Choi, T. H. Park, et al., The response characteristics of a gas sensor based on poly-3-hexylithiophene thin-film transistors, Sens. Actuators B: Chem. 146 (2010) 40-45.

[7] M. Ammam, B. Keita, L. Nadjo, J. Fransaer, pH sensor based on the crown heteropolyanion K28Li5H7P8W48O184.92 H2O immobilized using a layer by layer assembly process, Sens. Actuators B: Chem. 142 (2009) 347-354.

[8] A. Oprea, N. Bârsan, U. Weimar, M.-L. Bauersfeld, D. Ebling, J. Wöllenstein, Capacitive humidity sensors on flexible RFID labels, Sens. Actuators B: Chem. 132 (2008) 404-410.

[9] T. S. López, RFID and sensor integration standards: State and future prospects, Computer Standards \& Interfaces 33 (2011) 207-213.

[10] Boverket, God bebyggd miljö - förslag till nytt delmål för fukt och mögel: Resultat om byggnaders fuktskador från projektet BETSI,

http://www.boverket.se/Global/Webbokhandel/Dokument/2011/BETSI-Fukt-och-mogel.pdf, accessed November 2011.

[11] E. Traversa, Ceramic sensors for humidity detection: the state-of-the-art and future developments, Sens. Actuators B: Chem. 23 (1995) 135-156.

[12] M. Bayhan, N. Kavasoglu, A study on the humidity sensing properties of $\mathrm{ZnCr2O4-K2CrO4} \mathrm{ionic}$ conductive ceramic sensor, Sens. Actuators B: Chem. 117 (2006) 261-265.

[13] C. P. L. Rubinger, C. R. Martins, M. A. De Paoli, R. M. Rubinger, Sulfonated polystyrene polymer humidity sensor: Synthesis and characterization, Sens. Actuators B: Chem. 123 (2007) 42-49.

[14] A. Sun, L. Huang, Y. Li, Study on humidity sensing property based on TiO2 porous film and polystyrene sulfonic sodium, Sens. Actuators B: Chem. 139 (2009) 543-547.

[15] A. Sun, Y. Wang, Y. Li, Stability and water-resistance of humidity sensors using crosslinked and quaternized polyelectrolytes films, Sens. Actuators B: Chem. 145 (2010) 680-684.

[16] O. Larsson, E. Said, M. Berggren, X. Crispin, Insulator Polarization Mechanisms in Polyelectrolyte-Gated Organic Field-Effect Transistors, Adv. Funct. Mater. 19 (2009) 3334-3341.

[17] P. Andersson, R. Forchheimer, P. Tehrani, M. Berggren, Printable All-Organic Electrochromic Active-Matrix Displays, Adv. Funct. Mater. 17 (2007) 3074-3082.

[18] O. Larsson, X. Wang, M. Berggren, X. Crispin, Proton motion in a polyelectrolyte: A probe for wireless humidity sensors, Sens. Actuators B: Chem. 143 (2010) 482-486.

[19] K. G. Ong, C. A. Grimes, C. L. Robbins, R. S. Singh, Design and application of a wireless, passive, resonant-circuit environmental monitoring sensor, Sens. Actuators A: Phys. 93 (2001) 33-43.

[20] Acreo, Dry phase patterning, http://www.acreo.se/templates/Page__777.aspx, accessed November 2011

[21] WebShape AB, 2011, http://www.webshape.se/, SE0302467-6, accessed November 2011.

[22] DuPont, DuPont 7102 carbon conductive compositon, http://www2.dupont.com/MCM/en_US/assets/downloads/prodinfo/7102.pdf, accessed November 2011. 
Figures:

(a)

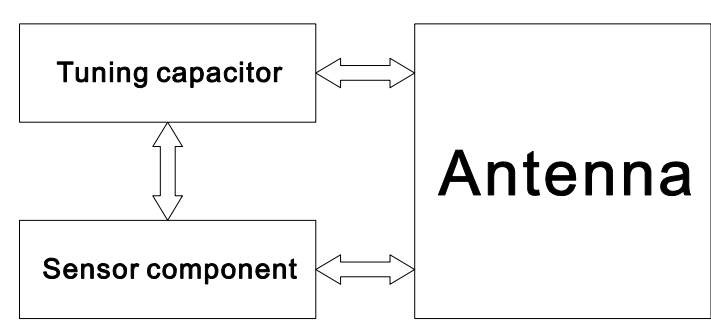

(b)

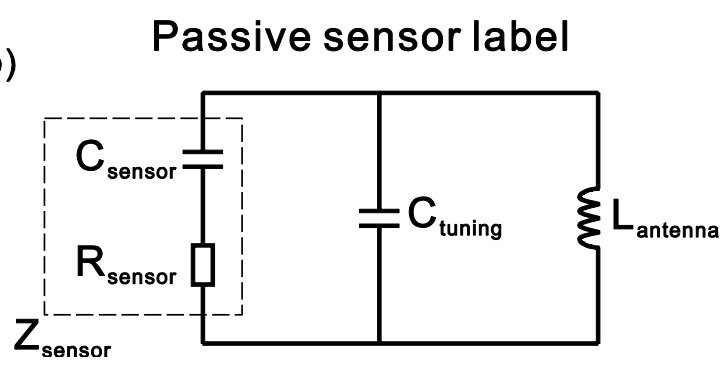

Fig. 1 (a) The function block diagram of the printed sensor label. (b) The electrical equivalent circuit of the printed sensor label. 


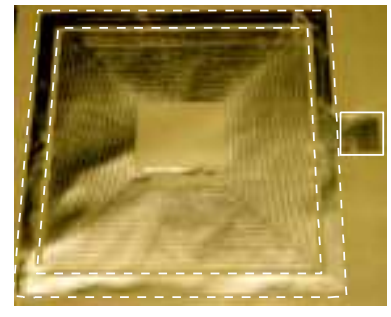

(a)

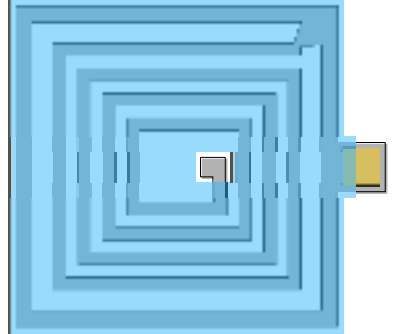

(c)

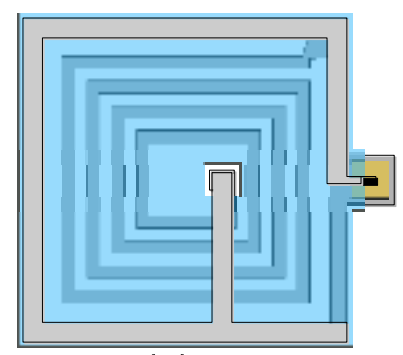

(e)

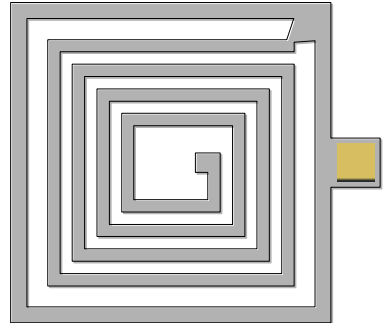

(b)

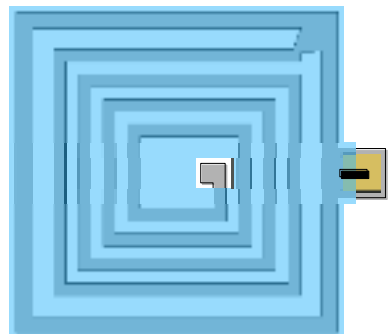

(d)

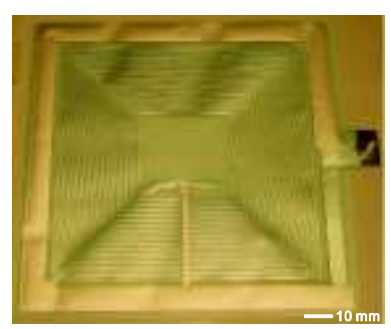

(f)

Fig. 2 (Color online) (a)-(e) Five steps of screen printing of a printed sensor label: (a) Photo of precleaned planar antenna and electrodes. (b) Screen printing of the electrolyte layer (yellow). (c) Screen printing of the dielectric layer (blue). (d) Screen printing of the top electrode of the printed sensor head (black). (e) Screen printing of the top electrode of the tuning capacitor and electrical bridges (light grey). (f) Photograph of an all-printed humidity sensor label. 


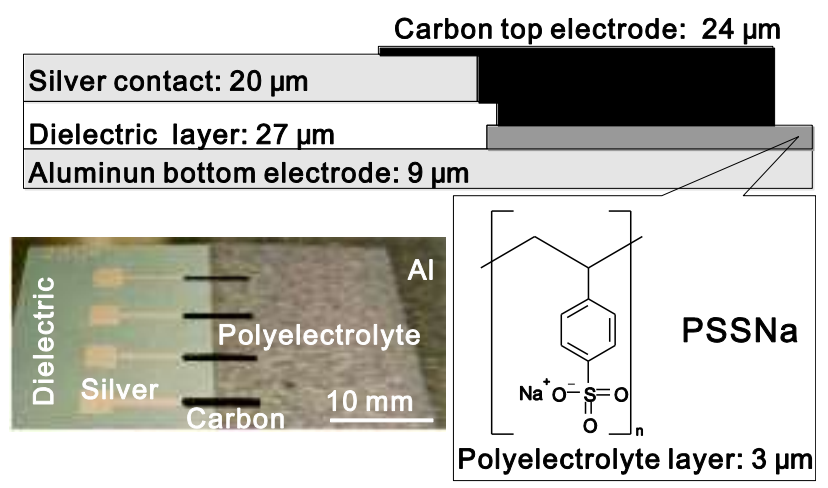

Fig. 3 The structure of a printed sensor head and a photo showing four sensor heads on a common bottom electrode. 
(a)

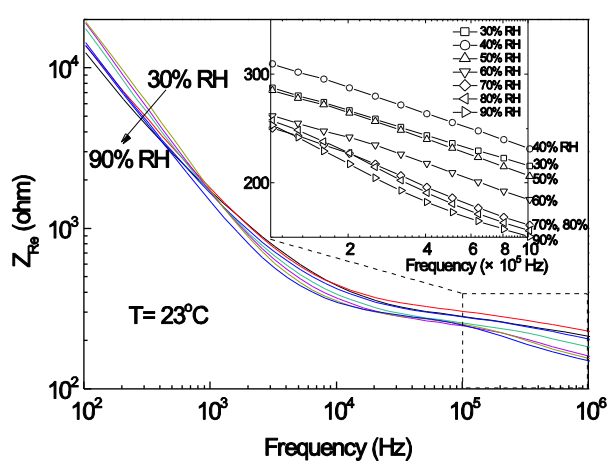

(b)

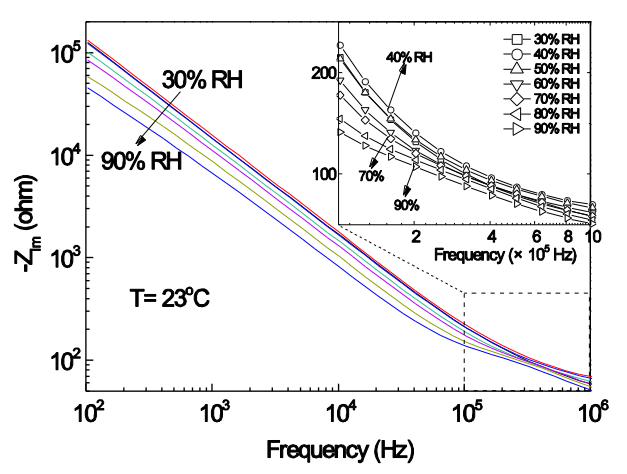

(c)

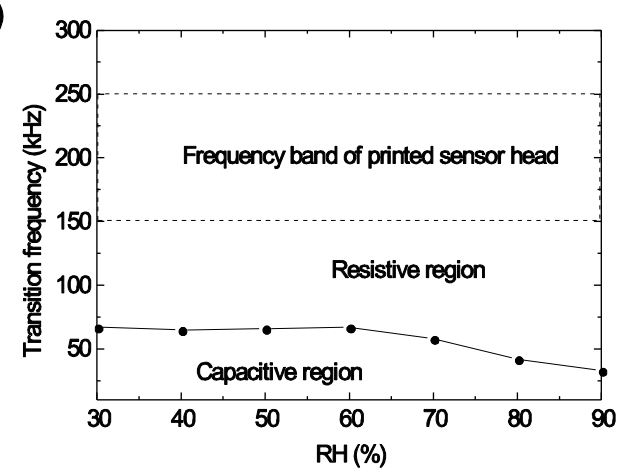

Fig. 4 (Color online) (a) The real part and (b) the imaginary part of the measured impedance of the printed senor head (area: $0.03 \mathrm{~cm}^{2}$ ). (c) The transition frequency versus relative humidity $(\mathrm{RH})$. 
(a)

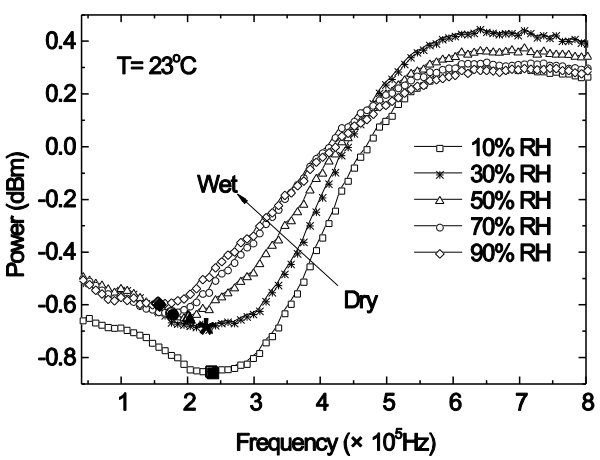

(b)

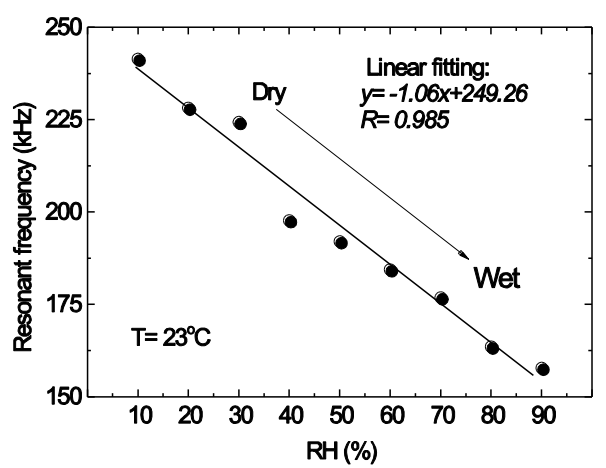

Fig. 5 (a) The evolution of the sensor label response at different humidity levels. (b) The resonant frequency of the printed sensor label versus relative humidity $(\mathrm{RH})$. 